\title{
Slicing Bing doubles
}

\author{
DAVID Cimasoni
}

\begin{abstract}
Bing doubling is an operation which produces a 2-component boundary link $B(K)$ from a knot $K$. If $K$ is slice, then $B(K)$ is easily seen to be boundary slice. In this paper, we investigate whether the converse holds. Our main result is that if $B(K)$ is boundary slice, then $K$ is algebraically slice. We also show that the Rasmussen invariant can tell that certain Bing doubles are not smoothly slice.
\end{abstract}

57M25; 57M27

\section{Introduction}

Bing doubling [2] is a standard construction which, given a knot $K$ in $S^{3}$, produces a 2-component oriented link $B(K)$ as illustrated below. (See Section 1 for a precise definition.)
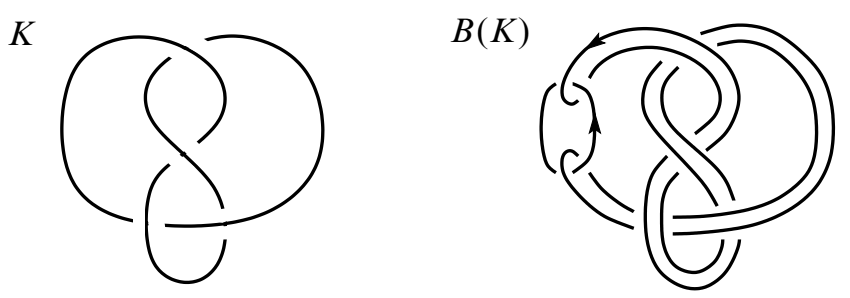

Figure 1: The figure eight knot and its Bing double

It is easy to check that if the knot $K$ is slice, then the link $B(K)$ is slice. Does the converse hold? An affirmative answer to this question seems out of reach. However, a result obtained independently by S Harvey [9] and P Teichner [22] provides a first step in this direction.

Recall that the Levine-Tristram signature of a knot $K$ is the function $\sigma_{K}: S^{1} \rightarrow \mathbb{Z}$ defined as follows: for $\omega \in S^{1}, \sigma_{K}(\omega)$ is given by the signature of the Hermitian matrix $(1-\omega) A+(1-\bar{\omega}) A^{*}$, where $A$ is a Seifert matrix for $K$ and $A^{*}$ denotes the transposed matrix. 
Theorem (Harvey [9], Teichner [22]) If $B(K)$ is slice, then the integral over $S^{1}$ of the Levine-Tristram signature of $K$ is zero.

For example, this shows that the Bing double of the trefoil knot is not slice. However, it says nothing about the Bing double of the figure eight knot (Figure 1), as $\sigma_{K}$ is identically zero. Let us also mention a conjecture of $\mathrm{R}$ Schneiderman and $\mathrm{P}$ Teichner: if $B(K)$ is slice, then the Arf invariant of $K$ is zero. They hope to prove this statement using tree-valued intersections of Whitney towers.

In this paper, we obtain a couple of results in the same direction. It is known that Bing doubles are boundary links: their components bound disjoint Seifert surfaces (see also Proposition 1.1). Furthermore, if a knot is slice, then its Bing double is not only slice, but boundary slice: the two slicing discs bound disjoint oriented 3-manifolds in the 4-ball (see also Proposition 1.2). Therefore, it makes sense to consider the following variation of the original question: is $B(K)$ boundary slice only when $K$ is slice ? Note that there is no known example of a boundary link which is slice and not boundary slice. Hence, it may turn out that both questions are in fact equivalent.

Using D Sheiham's work on boundary link concordance [20], we obtain the following result.

Theorem If $B(K)$ is boundary slice, then $K$ is algebraically slice.

This shows in particular that the Bing double of the figure eight knot is not boundary slice, a fact known to experts like K Ko [12].

In a final section, we show that the Rasmussen concordance invariant [17] (more specifically, its generalization to links due to A Beliakova and S Wehrli [1]) can be used to study the sliceness of Bing doubles. Indeed, we prove that if the ThurstonBennequin invariant of a knot $K$ is non-negative, then $s(B(K))=1$. Since the Rasmussen invariant of the 2-component unlink is -1 , it follows that such a Bing double is not smoothly slice. This latter statement can also be obtained from results of L Rudolph [19]. Nevertheless, our computations show that this fact follows very easily from elementary properties of the Rasmussen invariant. Note that there are examples of topologically slice knots with non-negative Thurston-Bennequin invariant (see Section 3). Bing doubling these knots gives the following result.

Theorem There exist links that are topologically slice but not smoothly slice, and whose components are trivial.

Algebraic 83 Geometric Topology, Volume 6 (2006) 
The paper is organized as follows. In Section 1, we give a precise definition of Bing doubling and recall some of its well-known properties. We also check that many 'classical' concordance invariants are useless for studying Bing doubles. (This is an attempt to convince the reader that the question under study is non-trivial). Section 2 starts with a brief survey of boundary link concordance theory, including Sheiham's [20]. We then prove our main result. In Section 3, we recall several properties of the Rasmussen invariant, and show that it can be used to detect some Bing doubles that are not smoothly slice.

\section{The Bing double of a knot, first properties}

Let $K$ be an oriented knot in $S^{3}$, and let $\mathcal{N}(K)$ denote a closed tubular neighborhood of $K$ in $S^{3}$. Recall that a pair $m, \ell$ of oriented simple closed curves in $\partial \mathcal{N}(K)$ is a standard meridian and longitude for $K$ if $[m]=0,[\ell]=[K]$ in $H_{1}(\mathcal{N}(K))$, and $l k(m, K)=1, l k(\ell, K)=0$, where $l k(\cdot, \cdot)$ denotes the linking number. Note that such a pair is unique up to isotopy.

Let $L$ denote the Borromean rings, arbitrarily oriented, and let $m_{0}, \ell_{0} \subset \partial \mathcal{N}\left(L_{0}\right)$ be a standard meridian and longitude for some component $L_{0}$ of $L$. The Bing double of $K$ is the 2-component oriented link $B(K)$ given by the image of $L \backslash L_{0}$ in $\left(S^{3} \backslash\right.$ int $\left.\mathcal{N}\left(L_{0}\right)\right) \cup\left(S^{3} \backslash\right.$ int $\left.\mathcal{N}(K)\right)=S^{3}$, where the pasting homomorphism maps $m$ onto $\ell_{0}$ and $\ell$ onto $m_{0}$. Figure 2 should convince the reader that this definition corresponds to the illustration given in Figure 1. Note that the isotopy type of the oriented link $B(K)$ depends neither on the choice of the component $L_{0}$ of $L$, nor on the orientation of $L$ and $K$. This follows from obvious symmetry properties of the Borromean rings.
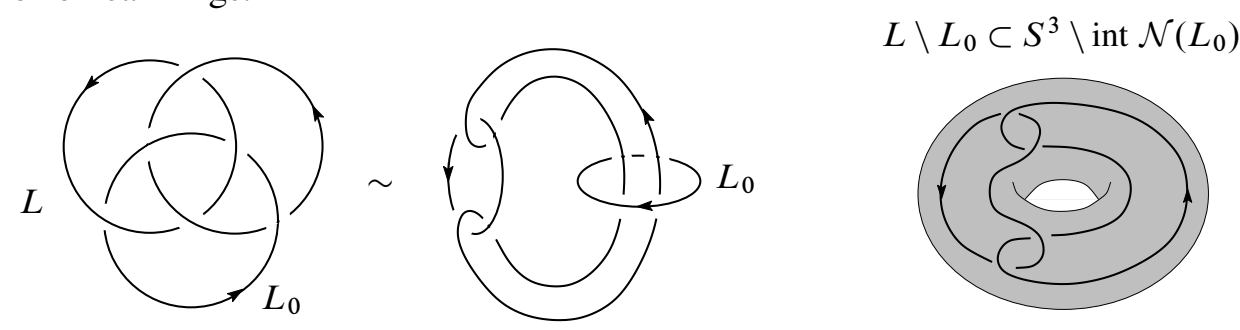

Figure 2: The Borromean rings $L$, and the solid torus $S^{3} \backslash \operatorname{int} \mathcal{N}\left(L_{0}\right)$

Recall that an oriented link is a boundary link if its components bound disjoint Seifert surfaces. The following seems well known.

Proposition 1.1 Bing doubles are boundary links. 
Proof Consider the pair $L_{1} \cup L_{2}:=L \backslash L_{0} \subset S^{3} \backslash$ int $\mathcal{N}\left(L_{0}\right)=S^{1} \times D^{2}$ illustrated in Figure 2, where the solid torus is parametrized using the standard meridian and longitude for $L_{0}$. Note that there are two disjoint genus 0 surfaces $P_{1}$ and $P_{2}$ in $S^{1} \times D^{2}$ such that the boundary of $P_{i}$ consists of $L_{i}$ together with two longitudes of the solid torus, for $i=1,2$. This is illustrated below.
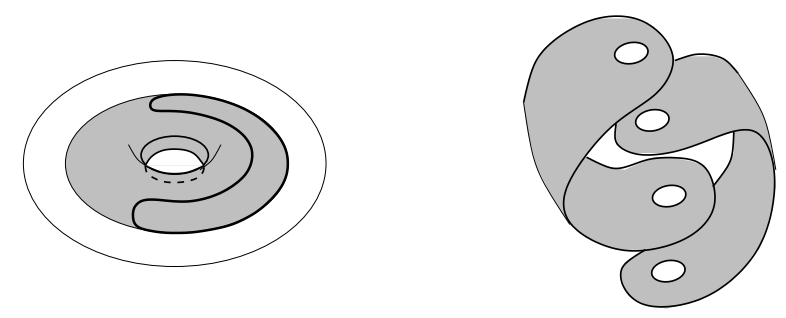

Figure 3: On the left, one of the $P_{i}$ 's in the solid torus. On the right, an illustration of how $P_{1}$ and $P_{2}$ are embedded with respect to each other.

The pasting homeomorphism $h: \partial\left(S^{1} \times D^{2}\right) \rightarrow \partial \mathcal{N}(K)$ maps the longitudes of $S^{1} \times D^{2}$ onto standard longitudes of $\mathcal{N}(K)$, that is, parallel unlinked copies of $K$. These parallel copies bound disjoint Seifert surfaces (parallel copies of a fixed Seifert surface for $K)$. Pasting $h\left(P_{1} \sqcup P_{2}\right)$ with 4 parallel Seifert surfaces for $K$, we obtain two disjoint Seifert surfaces for the components of $B(K)$.

Of course, if $K$ and $K^{\prime}$ are isotopic knots, then $B(K)$ and $B\left(K^{\prime}\right)$ are isotopic oriented links. Is the converse also true ? The answer is yes. Indeed, the Jaco-Shalen-Johannson decomposition theorem implies that two knots $K$ and $K^{\prime}$ are isotopic if and only if $B(K)$ and $B\left(K^{\prime}\right)$ are. We refer to Hatcher [10] for a beautiful exposition of the JSJ-decomposition theorem, and to Budney [3] for a survey of its consequences for knots and links in $S^{3}$. The fact mentioned above can be understood as a special case of [3, Proposition 4.31].

The aim of this paper is to address a 4-dimensional analogue of the question above. Recall that two $m$-component links $L_{0}$ and $L_{1}$ in $S^{3}$ are concordant if there is a proper oriented locally flat submanifold $C \subset S^{3} \times[0,1]$, homeomorphic to $m$ copies of $S^{1} \times[0,1]$, such that $C \cap S^{3} \times t=L_{t}$ for $t=0,1$. If these are boundary links and the concordance together with some disjoint Seifert surfaces on top and bottom bound $m$ disjoint oriented 3-manifolds in $S^{3} \times[0,1]$, then the links are boundary concordant. A (boundary) link is (boundary) slice if it is (boundary) concordant to the trivial link. Note that a knot is slice if and only if it is boundary slice. (Indeed, if $V$ is a Seifert surface for a knot that has a slicing disk $D$ in the 4-ball $B^{4}$, then elementary obstruction theory shows that $V \cup D$ bounds an oriented 3-manifold in $B^{4}$.) Note 
also that there is no known example of a boundary link which is slice and not boundary slice.

Proposition 1.2 If two knots $K$ and $K^{\prime}$ are concordant, then the links $B(K)$ and $B\left(K^{\prime}\right)$ are boundary concordant.

Proof Let $K$ and $K^{\prime}$ be concordant knots with Seifert surfaces $V$ and $V^{\prime}$. Fix a concordance $C: S^{1} \times[0,1] \hookrightarrow S^{3} \times[0,1]$ between $K$ and $K^{\prime}$, and let $W$ be an oriented 3-manifold in $S^{3} \times[0,1]$ such that $\partial W=V \cup C \cup-V^{\prime}$. Let $\widetilde{C}: S^{1} \times D^{2} \times$ $[0,1] \hookrightarrow S^{3} \times[0,1]$ be a parametrization of a tubular neighborhood of $C$, such that $\left(* \times \partial D^{2}, S^{1} \times *\right) \times t$ maps to a standard meridian and longitude for $K$ if $t=0$ and for $K^{\prime}$ if $t=1$. Finally, let $P$ be a pair of pants, and let $\varphi: P \sqcup P \hookrightarrow S^{1} \times D^{2}$ be a parametrization of the embedding illustrated in Figure 3. Consider the oriented 3-manifold $\widetilde{W}_{1} \sqcup \widetilde{W}_{2}$ given by the image of the embedding

$$
(P \sqcup P) \times[0,1] \stackrel{\varphi \times i d}{\longrightarrow} S^{1} \times D^{2} \times[0,1] \stackrel{\widetilde{C}}{\longrightarrow} S^{3} \times[0,1] .
$$

Note that for $i=1,2, \partial \widetilde{W}_{i}$ consists of a concordance between the $i^{\text {th }}$ component of $B(K)$ and $B\left(K^{\prime}\right)$, together with two parallel copies of $C$. Let $W_{i}$ denote the 3-manifold obtained by pasting $\widetilde{W}_{i}$ with two parallel copies of $W$. The disjoint 3-manifolds $W_{1}, W_{2}$ provide a boundary concordance between $B(K)$ and $B\left(K^{\prime}\right)$.

Corollary 1.3 If a knot $K$ is slice, then the link $B(K)$ is boundary slice.

This latter result is well-known to experts. It motivates the following:

Question If $B(K)$ is a slice link, is $K$ necessarily a slice knot?

To illustrate the difficulty of this problem, let us go through a list of obstructions to the sliceness of links. We shall see that all these obstructions vanish for all Bing doubles.

The (multivariable) Alexander polynomial It is well-known that the Alexander polynomial of a slice knot $K$ is of the form $\Delta_{K}(t)=f(t) f\left(t^{-1}\right)$ for some $f(t) \in \mathbb{Z}[t]$. A Kawauchi [11] generalized this result in the following way. Given an $m$-component ordered link $L$, let $\mathcal{A}(L)$ denote its Alexander module over $\mathbb{Z}\left[t_{1}^{ \pm 1}, \ldots, t_{m}^{ \pm 1}\right]$, and let $\widetilde{\Delta}_{L}$ be the greatest common divisor of the order ideal of the torsion of $\mathcal{A}(L)$. If $L$ is slice, then $\widetilde{\Delta}_{L}\left(t_{1}, \ldots, t_{m}\right)=f\left(t_{1}, \ldots, t_{m}\right) f\left(t_{1}^{-1}, \ldots, t_{m}^{-1}\right)$ for some $f\left(t_{1}, \ldots, t_{m}\right) \in$ $\mathbb{Z}\left[t_{1}, \ldots, t_{m}\right]$.

Let us now describe briefly how to compute the Alexander module of a Bing double, referring to Cooper [6] and Cimasoni-Florens [5] for details. Recall that a C-complex 
for a 2-component link $L=L_{1} \cup L_{2}$ is given by two Seifert surfaces $V_{1}$ for $L_{1}$ and $V_{2}$ for $L_{2}$ that intersect only along clasps. Given such a C-complex $V=V_{1} \cup V_{2}$, one can define two 'generalized Seifert forms' on $H_{1}(V)$, giving two Seifert matrices, say $A$ and $A^{\prime}$. A presentation matrix for $\mathcal{A}(L)$ can be obtained by some $\mathbb{Z}\left[t_{1}^{ \pm 1}, t_{2}^{ \pm 1}\right]-$ linear combination of these matrices and their transpose. This computational method is very efficient in the case of a Bing double $L=B(K)$. Indeed, there is an obvious C-complex $V$ for $B(K)$ given by two discs intersecting along two clasps. Then, $H_{1}(V)=\mathbb{Z}$, and since the Bing double is untwisted, we get $A=A^{\prime}=(0)$. Therefore, $\mathcal{A}(B(K))=\mathbb{Z}\left[t_{1}^{ \pm 1}, t_{2}^{ \pm 1}\right]$ for any knot $K$, so $B(K)$ has the Alexander module of a trivial link. In particular, $\widetilde{\Delta}_{B(K)}\left(t_{1}, t_{2}\right)=1$ for all knots $K$.

The (multivariable) Levine-Tristram signature The Levine-Tristram signature of a knot $K$ is the function $\sigma_{K}: S^{1} \rightarrow \mathbb{Z}$ defined as follows: for $\omega \in S^{1}, \sigma_{K}(\omega)$ is given by the signature of the Hermitian matrix $(1-\omega) A+(1-\bar{\omega}) A^{*}$, where $A$ is a Seifert matrix for $K$. If $K$ is a slice knot, then $\sigma_{K}(\omega)=0$ for all $\omega \in S^{1}$ such that $\Delta_{K}(\omega) \neq 0$. This invariant admits a multivariable generalization: for a 2-component ordered link $L$, it consists of a function $\sigma_{L}: S^{1} \times S^{1} \rightarrow \mathbb{Z}$. The integer $\sigma_{L}\left(\omega_{1}, \omega_{2}\right)$ is the signature of a Hermitian matrix given by some $\mathbb{C}$-linear combination (depending on $\omega_{1}$ and $\left.\omega_{2}\right)$ of the Seifert matrices $A$ and $A^{\prime}$ for $L$. If $L$ is a slice link, then $\sigma_{L}\left(\omega_{1}, \omega_{2}\right)=0$ for all $\omega_{1}, \omega_{2} \in S^{1}$ such that $\widetilde{\Delta}_{L}\left(\omega_{1}, \omega_{2}\right) \neq 0$. (Here again, we refer to [6] for the case of 2-component links, and to [5] for the general case.)

As mentioned in the previous paragraph, there is a choice of C-complex for $L=B(K)$ such that $A=A^{\prime}=(0)$. Therefore, $\sigma_{B(K)}=0$ for all knots $K$, so this invariant does not tell us anything about Bing doubles.

Remark In the construction of the Bing double of $K$, if one replaces the standard longitude for $K$ by some longitude that links $K$ (say, $t$ times), then the result is the $t$-twisted Bing double $B(K, t)$. One easily checks that $\sigma_{B(K, t)}\left(\omega_{1}, \omega_{2}\right)$ is equal to the sign of $t$ for all $\omega_{1}, \omega_{2} \neq 1$. Hence, the $t$-twisted Bing double of a knot is never slice if $t \neq 0$ (unlike some $t$-twisted Whitehead doubles). This is the reason why we restrict ourselves to the study of untwisted Bing doubles.

The Arf invariant Let $\alpha_{2}: H_{1}\left(V ; \mathbb{Z}_{2}\right) \times H_{1}\left(V ; \mathbb{Z}_{2}\right) \rightarrow \mathbb{Z}_{2}$ denote the Seifert form of a knot $K$ reduced modulo 2. The Arf invariant $\operatorname{Arf}(K)$ of $K$ is defined as the Arf invariant of the non-singular quadratic form $q: H_{1}\left(V ; \mathbb{Z}_{2}\right) \rightarrow \mathbb{Z}_{2}$ given by $q(x)=\alpha_{2}(x, x)$. If $K$ is a slice knot, then $\operatorname{Arf}(K)=0$. If $L$ is an oriented link with components $\left\{L_{i}\right\}$ that satisfy $l k\left(L_{i}, L \backslash L_{i}\right) \equiv 0(\bmod 2)$ for all $i$, then its reduced Seifert form 
$\alpha_{2}$ induces a well-defined non-singular quadratic form on $H_{1}\left(V ; \mathbb{Z}_{2}\right) / i_{*} H_{1}\left(\partial V ; \mathbb{Z}_{2}\right)$. The Arf invariant of $L$ is then defined as the Arf invariant of this quadratic form.

The Bing double of a knot obviously satisfies the condition above and not surprisingly, $\operatorname{Arf}(B(K))=0$ for all knots $K$. There are numerous ways to check this fact. For example, K Murasugi [16] showed that if $L=L_{1} \cup L_{2}$ satisfies $l k\left(L_{1}, L_{2}\right) \equiv 0$ $(\bmod 2)$, then

$$
\operatorname{Arf}(L) \equiv \operatorname{Arf}\left(L_{1}\right)+\operatorname{Arf}\left(L_{2}\right)+\left.\frac{1}{2} \frac{d^{2}}{d t^{2}} \Delta_{L}(t, t)\right|_{t=1}(\bmod 2) .
$$

In the case of $L=B(K)$, both components are trivial and $\Delta_{L}\left(t_{1}, t_{2}\right)=0$, leading to the result.

The Milnor $\bar{\mu}$-invariants As we saw in Proposition $1.1, B(K)$ is a boundary link. This implies the vanishing of another obstruction to the sliceless of $B(K)$ : Milnor's $\bar{\mu}$-invariants.

The slice Bennequin inequality Up to now, we have been concerned with the topological concordance of links. If one requires the concordance to be smooth, one gets a stronger equivalence relation: there are links which are topologically slice (bound disjoint locally flat discs in $B^{4}$ ) but not smoothly slice (do not bound disjoint smooth discs in $B^{4}$ ). We shall now investigate an obstruction to the smooth sliceness of links. Given an oriented link $L$, let $\chi_{s}(L)$ denote the greatest Euler characteristic $\chi(F)$ of an oriented surface $F$ (with no closed component) smoothly embedded in $B^{4}$ with boundary $L$. The slice Bennequin inequality (Rudolph [18]) asserts that for every braid $\beta \in B_{n}$,

$$
\chi_{s}(\widehat{\beta}) \leq n-\omega(\beta),
$$

where $\hat{\beta}$ denotes the closure of $\beta$ and $\omega(\beta)$ its writhe (ie, the number of positive crossings minus the number of negative ones).

This inequality does not tell us anything about Bing doubles. Indeed, let $L=B(K)=$ $L_{1} \cup L_{2}$ and fix $\beta \in B_{n}$ such that $\hat{\beta}=L$. Write the geometric braid $\beta$ as a union $\beta=\beta_{1} \cup \beta_{2}$ with $\hat{\beta}_{i}=L_{i}$ and $\beta_{i} \in B_{n_{i}}$ for $i=1$, 2. Clearly, $n_{1}+n_{2}=n$ and $\omega(\beta)=\omega\left(\beta_{1}\right)+\omega\left(\beta_{2}\right)+2 l k\left(L_{1}, L_{2}\right)=\omega\left(\beta_{1}\right)+\omega\left(\beta_{2}\right)$. Since each $L_{i}$ is the unknot, the slice Bennequin inequality applied to $\beta_{i}$ gives $1 \leq n_{i}-\omega\left(\beta_{i}\right)$. Summing over $i=1,2$ gives $2 \leq n-\omega(\beta)$. This means that, in the best case, the slice Bennequin inequality for $\beta$ will read $\chi_{s}(L) \leq 2$. This does not say anything about $L$, as this inequality holds for any 2 -component link.

To conclude this panorama, it should be mentioned that invariants of Cha-Ko [4] and Friedl [8] do detect the Bing double of some knots with non-trivial Levine-Tristram 
signature. This is also true for the $L^{2}$-signatures of Harvey [9], as mentioned in the introduction. However, none of these invariants can detect the Bing double of a torsion element in the knot concordance group.

\section{Boundary sliceness of Bing doubles}

Since Bing doubles are boundary links, and since $B(K)$ is boundary slice whenever $K$ is slice, it makes sense to consider the following variation of our original problem:

Question If $B(K)$ is boundary slice, is $K$ necessarily slice?

Let us recall once again that there is no known example of a boundary link which is slice and not boundary slice. Therefore, both questions might turn out to be equivalent. Nevertheless, we shall be more successful with this version.

In order to state our results, let us recall several standard facts about boundary link concordance. We refer to Ko [13] for proofs and further details.

Let $B(m)$ denote the set of boundary concordance classes of $m$-component boundary links in $S^{3}$. A pair $(L, V)$ consisting of a boundary link $L=L_{1} \cup \cdots \cup L_{m}$ together with disjoint Seifert surfaces $V=V_{1} \cup \cdots \cup V_{m}$ is called a boundary pair. Two boundary pairs $(L, V)$ and $\left(L^{\prime}, V^{\prime}\right)$ are said to be concordant if there are disjoint oriented 3-manifolds $W_{1}, \ldots, W_{m}$ in $B^{4}$ such that $\partial W_{i}=V_{i} \cup C_{i} \cup-V_{i}^{\prime}$, where $C_{i}$ is a concordance between $L_{i}$ and $L_{i}^{\prime}$. The set of concordance classes of boundary pairs is denoted by $C\left(B_{m}\right)$. Then, the obvious surjective map $C\left(B_{m}\right) \rightarrow B(m)$ turns out to be a bijection for $m \leq 2$. (This is false for $m>2$.)

Fix an $m$-component boundary pair $(L, V)$. For $i, j=1, \ldots, m$, consider the bilinear pairing $H_{1}\left(V_{i}\right) \times H_{1}\left(V_{j}\right) \rightarrow \mathbb{Z}$ defined by $(x, y) \mapsto l k\left(x^{+}, y\right)$, where $x^{+}$denotes the 1-cycle $x$ pushed in the positive normal direction of $V_{i}$. Let $A_{i j}$ denote the matrix of this pairing with respect to some fixed bases of $H_{1}\left(V_{i}\right)$ and $H_{1}\left(V_{j}\right)$. Note that $A_{i j}=A_{j i}^{*}$ if $i \neq j$ and $A_{i i}-A_{i i}^{*}$ is nothing but the intersection matrix of $V_{i}$. Furthermore, if the boundary pair $(L, V)$ is slice, then for suitable bases of the $H_{1}\left(V_{i}\right)$ 's, each $A_{i j}$ is metabolic: the upper left quadrant is zero. In this case, the collection $A=\left\{A_{i j}\right\}$ (and the boundary pair $\left.(L, V)\right)$ are said to be algebraically slice. This motivates the following definition: consider the set of collections $A=\left\{A_{i j}\right\}_{i, j=1}^{m}$

of integral matrices such that $A_{i j}=A_{j i}^{*}$ if $i \neq j$ and $A_{i i}-A_{i i}^{*}$ is unimodular. Let us say that two such collections $A$ and $B$ are equivalent if the block sum $A \oplus(-B)$ defined by $(A \oplus(-B))_{i j}=A_{i j} \oplus\left(-B_{i j}\right)$ is algebraically slice. Then, the set of equivalence classes forms an abelian group $G^{m}(\mathbb{Z})$ under the block sum. 
The Seifert matrix construction described above defines a map $\psi_{m}: C\left(B_{m}\right) \rightarrow G^{m}(\mathbb{Z})$ which is onto but not injective. Recall that there are canonical bijections $C\left(B_{1}\right) \cong$ $B(1) \cong \mathcal{C}$ and $C\left(B_{2}\right) \cong B(2)$, where $\mathcal{C}$ is the classical knot concordance group. Therefore, we have an epimorphism $\psi_{1}: \mathcal{C} \rightarrow G^{1}(\mathbb{Z})$ and a surjective map $\psi_{2}: B(2) \rightarrow$ $G^{2}(\mathbb{Z})$.

We are finally ready to state our main result.

Theorem 2.1 (i) The Bing doubling operation induces a map $\mathcal{C} \rightarrow B(2)$ and a homomorphism $\varphi: G^{1}(\mathbb{Z}) \rightarrow G^{2}(\mathbb{Z})$ such that the following diagram commutes:

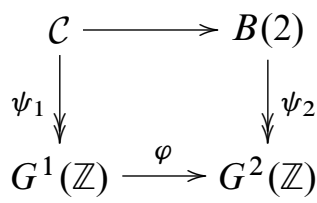

(ii) The homomorphism $\varphi$ is injective.

Corollary 2.2 If $B(K)$ is boundary slice, then $K$ is algebraically slice.

Proof of (i) The fact that Bing doubling induces a map $\mathcal{C} \rightarrow B(2)$ follows from Proposition 1.2. Let us define the homomorphism $\varphi: G^{1}(\mathbb{Z}) \rightarrow G^{2}(\mathbb{Z})$. Given a matrix $A$ such that $A-A^{*}$ is unimodular, set

$$
B(A)=\left(\begin{array}{ll}
A_{11} & A_{12} \\
A_{21} & A_{22}
\end{array}\right)
$$

where:

$$
A_{11}=A_{22}=\left(\begin{array}{ll}
A & A \\
A^{*} & A^{*}
\end{array}\right) \quad \text { and } \quad A_{12}=A_{21}^{*}=\left(\begin{array}{ll}
A & A \\
A^{*} & A
\end{array}\right)
$$

Note that $A_{12}=A_{21}^{*}$ and that $A_{11}-A_{11}^{*}=A_{22}-A_{22}^{*}=\left(\begin{array}{cc}A-A^{*} & 0 \\ 0 & A-A^{*}\end{array}\right)$ is unimodular since $A-A^{*}$ is. Hence, $B(A)$ defines an element of the group $G^{2}(\mathbb{Z})$. Let us now assume that $A$ is algebraically slice, say of size $2 g$. This means that there exists some unimodular matrix $Q$ of size $2 g$ such that $Q A Q^{*}$ is metabolic. Consider the unimodular matrix

$$
\widehat{Q}=\left(\begin{array}{cccc}
I_{g} & 0 & 0 & 0 \\
0 & 0 & I_{g} & 0 \\
0 & I_{g} & 0 & 0 \\
0 & 0 & 0 & I_{g}
\end{array}\right)\left(\begin{array}{cc}
Q & 0 \\
0 & Q
\end{array}\right)
$$

Algebraic 83 Geometric Topology, Volume 6 (2006) 
where $I_{g}$ denotes the identity matrix of size $g$. A straightforward computation shows that $\widehat{Q} A_{i j} \widehat{Q}^{*}$ is metabolic for all $i, j$. Hence, $B(A)$ is algebraically slice. Now, let $A$ and $A^{\prime}$ be two Seifert matrices of respective size $2 g$ and $2 g^{\prime}$. Consider the unimodular matrix:

$$
R=\left(\begin{array}{cccc}
I_{2 g} & 0 & 0 & 0 \\
0 & 0 & I_{2 g} & 0 \\
0 & I_{2 g^{\prime}} & 0 & 0 \\
0 & 0 & 0 & I_{2 g^{\prime}}
\end{array}\right)
$$

One checks that

$$
\left(\begin{array}{ll}
R & 0 \\
0 & R
\end{array}\right) B\left(A \oplus A^{\prime}\right)\left(\begin{array}{cc}
R^{*} & 0 \\
0 & R^{*}
\end{array}\right)=B(A) \oplus B\left(A^{\prime}\right) .
$$

These facts imply that $A \mapsto B(A)$ defines a homomorphism $\varphi: G^{1}(\mathbb{Z}) \rightarrow G^{2}(\mathbb{Z})$. It remains to check that the diagram commutes. Let $(K, V)$ be a 1-component boundary pair (that is, a knot with a Seifert surface), and let $A$ denote the associated Seifert matrix with respect to some basis $b$ of $H_{1}(V)$. Let $B(V)=B(V)_{1} \sqcup B(V)_{2}$ denote the boundary Seifert surface for $B(K)$ constructed in the proof of Proposition 1.1. Clearly, $H_{1}\left(B(V)_{1}\right)=H_{1}\left(B(V)_{2}\right)=H_{1}(V) \oplus H_{1}(V)$. Consider the right hand side of Figure 3: the four glued in Seifert surfaces are parallel, but their orientations alternate. It follows that the Seifert matrix with respect to the basis $b \cup b$ of $H_{1}\left(B(V)_{1}\right)$ and $H_{1}\left(B(V)_{2}\right)$ is given by $B(A)$.

Our proof the injectivity of $\varphi$ makes extensive use of D Sheiham's interpretation of $G^{m}(\mathbb{Z})$ as the Witt group of the representation category of some ring $P_{m}$. We shall now quickly review the notions and results involved, referring to [20] for further details and proofs.

Fix a commutative ring $\Lambda$ and a ring $R$ with an involution $r \mapsto \bar{r}$. Let $(R-\Lambda)-$ Proj denote the category of representations $\rho: R \rightarrow \operatorname{End}_{\Lambda}(M)$, where $M$ is a finitely generated projective $\Lambda$-module. This is a Hermitian category via $(M, \rho) \mapsto\left(M^{*}, \rho^{*}\right)$, where $M^{*}=\operatorname{Hom}_{\Lambda}(M, \Lambda)$ and $\rho^{*}: R \rightarrow \operatorname{End}_{\Lambda}\left(M^{*}\right)$ is given by $\rho^{*}(r)(\xi): x \mapsto$ $\xi(\rho(\bar{r})(x))$ for $r \in R, \xi \in M^{*}$ and $x \in M$.

Fix a sign $\epsilon= \pm 1$. An $\epsilon$-hermitian form $(M, \phi)$ is a morphism $\phi: M \rightarrow M^{*}$ in $(R-\Lambda)-$ Proj such that $\phi^{*}=\epsilon \phi$. (Here, $\left(M^{*}\right)^{*}$ is identified with $M$.) An object $M$ is $\epsilon$-self dual if there exists an $\epsilon$-hermitian form $(M, \phi)$ which is non-singular, ie an isomorphism. Finally, recall that an $\epsilon$-hermitian form $(M, \phi)$ is called metabolic if there is a direct summand $j: L \hookrightarrow M$ such that $L=\operatorname{ker}\left(j^{*} \phi: M \rightarrow L^{*}\right)$. The Witt group of the Hermitian category $(R-\Lambda)$ - Proj is the abelian group $W^{\epsilon}(R-\Lambda)$ with 
one generator $[M, \phi]$ for each isomorphism class of non-singular $\epsilon$-hermitian form $(M, \phi)$ subject to the relations

$$
\begin{cases}{\left[M^{\prime}, \phi^{\prime}\right]=[M, \phi]+\left[M^{\prime \prime}, \phi^{\prime \prime}\right]} & \text { if }\left(M^{\prime}, \phi^{\prime}\right) \cong(M, \phi) \oplus\left(M^{\prime \prime}, \phi^{\prime \prime}\right) ; \\ {[M, \phi]=0} & \text { if }(M, \phi) \text { is metabolic. }\end{cases}
$$

Note that $W^{\epsilon}(\mathbb{Z}-\Lambda)$ is simply the Witt group $W^{\epsilon}(\Lambda)$ of the ring $\Lambda$.

Let us now focus on some particular choice of the ring $R$. Let $m \geq 1$ be an integer, and let $P_{m}$ be the ring

$$
\left.P_{m}=\mathbb{Z}\left\langle s, \pi_{1}, \ldots, \pi_{m}\right| \pi_{i}^{2}=\pi_{i}, \pi_{i} \pi_{j}=0 \text { for } i \neq j, \sum_{i=1}^{m} \pi_{i}=1\right\rangle
$$

endowed with the involution induced by $s \mapsto \bar{s}=1-s$ and $\pi_{i} \mapsto \overline{\pi_{i}}=\pi_{i}$ for $i=1, \ldots, m$. Given any commutative ring $\Lambda$, Sheiham's idea is to interpret the group $G^{m}(\Lambda)$ as the Witt group $W^{-1}\left(P_{m}-\Lambda\right)$. Indeed, an element in $G^{m}(\Lambda)$ given by a collection of matrices $A=\left\{A_{i j}\right\}_{i, j=1}^{m}$ can be understood as the equivalence class of an $(m+2)$-tuple $\left(M, \pi_{1}, \ldots, \pi_{m}, \lambda\right)$, where $M$ is a finitely generated projective $\Lambda$-module, $\pi_{1}, \ldots, \pi_{m}$ is a set of orthogonal idempotents in $\operatorname{End}_{\Lambda}(M)$ and $\lambda: M \rightarrow$ $M^{*}$ is an $\Lambda$-module homomorphism such that $\lambda-\lambda^{*}$ is an isomorphism which commutes with each $\pi_{i}$. ( $A_{i j}$ should be thought of as the matrix of a homomorphism $\pi_{i} M \rightarrow\left(\pi_{j} M\right)^{*}$, where the $\pi_{i} M$ 's are free $\Lambda$-modules; the collection of matrices $A$ then defines a homomorphism $\lambda: M \rightarrow M^{*}$, where $M=\bigoplus_{i=1}^{m} \pi_{i} M$.) Given such an $(m+2)$-tuple, set $\phi=\lambda-\lambda^{*}$ and define $\rho: P_{m} \rightarrow \operatorname{End}_{\Lambda}(M)$ by $\rho(s)=\phi^{-1} \lambda$, $\rho\left(\pi_{i}\right)=\pi_{i}$. One easily checks that $(M, \phi)$ is a non-singular $(-1)$-hermitian form. The only non-trivial point is the equality $\phi(s x)(y)=\phi(x)(\bar{s} y)$ for $x, y \in M$, where $s x$ stands for $\rho(s)(x)$. It follows from the following equations:

$$
\begin{aligned}
\phi(s x)(y)+\phi(x)(s y) & =\phi \phi^{-1} \lambda(x)(y)+\phi(x)\left(\phi^{-1} \lambda(y)\right) \\
& =\lambda(x)(y)+\lambda^{*}\left(\phi^{-1}\right)^{*} \phi(x)(y)=\left(\lambda-\lambda^{*}\right)(x)(y)=\phi(x)(y) .
\end{aligned}
$$

It turns out that this construction induces an isomorphism

$$
G^{m}(\Lambda) \stackrel{\kappa}{\longrightarrow} W^{-1}\left(P_{m}-\Lambda\right), \quad\left[M, \pi_{1}, \ldots, \pi_{m}, \lambda\right] \longmapsto[M, \phi]
$$

which is natural with respect to $\Lambda$ (see [20, Lemma 3.31]).

The next step makes use of so-called Hermitian devissage. For the Hermitian category $(R-k)-$ Proj, where $k$ is a field, it can be stated as follows. Let $\overline{\mathcal{M}}^{s}(R-k, \epsilon)$ denote the set of isomorphism classes of simple $\epsilon$-self-dual objects in $(R-k)-$ Proj. Then, 
there is a canonical isomorphism of Witt groups

$$
W^{\epsilon}(R-k) \cong \bigoplus_{M \in \overline{\mathcal{M}}^{s}(R-k, \epsilon)} W^{\epsilon}\left(\left.(R-k)\right|_{M}\right),
$$

where $\left.(R-k)\right|_{M}$ denotes the (Hermitian) full subcategory of $(R-k)$ - Proj whose objects are isomorphic to a direct summand of $M^{\oplus d}$ for some $d$.

We conclude this brief exposition of Sheiham's work with the following special case of Hermitian Morita equivalence. Fix a non-singular $\epsilon$-hermitian form $(M, b)$ in $(R-k)-$ Proj. Then, we have an isomorphism

$$
\begin{aligned}
& W^{\epsilon}\left(\left.(R-k)\right|_{M}\right) \stackrel{\Theta_{M, b}}{\longrightarrow} W^{1}\left(\operatorname{End}_{(R-k)} M\right) \\
& {[N, \phi] \longmapsto\left[\operatorname{Hom}(M, N), \Phi_{N} \phi_{*}\right],}
\end{aligned}
$$

where $\operatorname{Hom}(M, N)=\operatorname{Hom}_{(R-k)}(M, N)$ is endowed with the obvious structure of right $\operatorname{End}_{(R-k)} M$-module, and $\Phi_{N} \phi_{*}: \operatorname{Hom}(M, N) \rightarrow \operatorname{Hom}(M, N)^{*}$ is given by $\left(\Phi_{N} \phi_{*}\right)(\alpha)(\beta)=\epsilon b^{-1} \alpha^{*} \phi \beta$ for $\alpha, \beta \in \operatorname{Hom}(M, N)$.

Finally, note that the natural homomorphism $G^{m}(\mathbb{Z}) \rightarrow G^{m}(\mathbb{Q})$ is injective [20, Lemma 1.11]. Summing up, we have the following sequence of homomorphisms:

$$
\begin{aligned}
G^{m}(\mathbb{Z}) & \hookrightarrow G^{m}(\mathbb{Q}) \stackrel{\kappa}{\longrightarrow} W^{-1}\left(P_{m}-\mathbb{Q}\right) \cong \bigoplus_{M} W^{-1}\left(\left.\left(P_{m}-\mathbb{Q}\right)\right|_{M}\right) \\
& \stackrel{p_{M}}{\rightarrow} W^{-1}\left(\left.\left(P_{m}-\mathbb{Q}\right)\right|_{M}\right) \stackrel{\Theta_{M, b}}{\longrightarrow} W^{1}\left(\operatorname{End}_{\left(P_{m}-\mathbb{Q}\right)} M\right),
\end{aligned}
$$

where the direct sum is over all $M \in \overline{\mathcal{M}}^{S}\left(P_{m}-\mathbb{Q},-1\right)$, and $p_{M}$ denotes the canonical projection corresponding to $M$.

Proof of (ii) We shall now use these results to prove the second part of Theorem 2.1. Recall that the Bing doubling map $\varphi: G^{1}(\mathbb{Z}) \rightarrow G^{2}(\mathbb{Z})$ (and similarly, the induced map $G^{1}(\mathbb{Q}) \rightarrow G^{2}(\mathbb{Q})$ ) is given by $[A] \mapsto[B(A)]=\left[\begin{array}{ll}A_{11} & A_{12} \\ A_{21} & A_{22}\end{array}\right]$, where the $A_{i j}$ 's are given by (1). This matrix $B(A)$ is block-congruent to (and therefore, represents the same class as) the matrix

$$
\widehat{A}:=\left(\begin{array}{cccc}
0 & T & T & 0 \\
0 & A^{*} & T^{*} & A \\
T^{*} & T & 0 & T \\
0 & A^{*} & 0 & A^{*}
\end{array}\right),
$$

where the letter $T$ stands for the unimodular matrix $A-A^{*}$. The isomorphism $\kappa: G^{1}(\mathbb{Q}) \rightarrow W^{-1}\left(P_{1}-\mathbb{Q}\right)$ maps $[A]$ to $[M, \phi]$, where $M$ is the $\mathbb{Q}$-vector space acted 
on by $A, \phi: M \rightarrow M^{*}$ is given by the matrix $T=A-A^{*}$, and the action of $P_{1}=\mathbb{Z}[s]$ on $M$ is defined by the matrix $S=T^{-1} A$. Similarly, $\kappa: G^{2}(\mathbb{Q}) \rightarrow W^{-1}\left(P_{2}-\mathbb{Q}\right)$ maps $[\widehat{A}]$ to $[\widehat{M}, \widehat{\phi}]$, where $\widehat{M}=M^{\oplus 4}$ as a $\mathbb{Q}$-vector space, $\widehat{\phi}: \widehat{M} \rightarrow(\widehat{M})^{*}$ is given by the matrix

$$
\widehat{T}:=\widehat{A}-(\widehat{A})^{*}=\left(\begin{array}{cccc}
0 & T & 0 & 0 \\
T & T^{*} & 0 & 0 \\
0 & 0 & 0 & T \\
0 & 0 & T & T^{*}
\end{array}\right)
$$

and the $P_{2}$-action on $\widehat{M}$ is defined as follows: for $x=\left(x_{1}, x_{2}, x_{3}, x_{4}\right) \in M^{\oplus 4}=\widehat{M}$, $\pi_{1} x=\left(x_{1}, x_{2}, 0,0\right), \pi_{2} x=\left(0,0, x_{3}, x_{4}\right)$, and $s x=\widehat{S} x$, where:

$$
\widehat{S}=(\widehat{T})^{-1} \widehat{A}=\left(\begin{array}{cccc}
0 & S & 0 & S \\
0 & I & I & 0 \\
-I & S & 0 & S \\
-I & I & 0 & I
\end{array}\right)
$$

With these notations, we have the commutative diagram

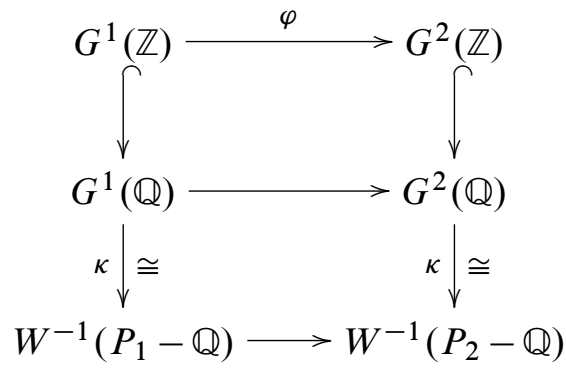

where the bottom homomorphism is given by $[M, \phi] \mapsto[\widehat{M}, \widehat{\phi}]$ as described above.

From now on, let us denote by $\mathcal{C}$ and $\mathcal{D}$ the Hermitian categories $\left(P_{1}-\mathbb{Q}\right)-$ Proj and $\left(P_{2}-\mathbb{Q}\right)-$ Proj, respectively.

Lemma 2.3 If $M$ is a simple object in $\mathcal{C}$, then $\widehat{M}$ is a simple object in $\mathcal{D}$.

Proof First note that if $M$ is a simple representation over $\mathbb{Q}$ of $P_{1}=\mathbb{Z}[s]$, then $M=\mathbb{Q}[s] /(p)$ with $p \in \mathbb{Q}[s]$ some irreducible polynomial in $\mathbb{Q}[s]$. If $S$ denotes a matrix of the action of $s \in \mathbb{Z}[s]$ on $M$ viewed as a $\mathbb{Q}$-vector space, then its characteristic polynomial $\operatorname{det}(S-s I)$ is equal to $p(s)$ up to multiplication by a non-zero scalar. Therefore, $\operatorname{det}(S-s I)$ must be irreducible in $\mathbb{Q}[s]$.

Let $n$ denote the dimension of $M$ over $\mathbb{Q}$, and let us assume that there is a $P_{2}-$ submodule $N$ of $\widehat{M}$ with $N \neq 0$ and $N \neq \widehat{M}$. In particular, the $\mathbb{Q}$-vector space 
$\widehat{M}=\pi_{1} \widehat{M} \oplus \pi_{2} \widehat{M}$ can be further decomposed into $\pi_{1} \widehat{M}=\pi_{1} N \oplus N_{1}^{\prime}$ and $\pi_{2} \widehat{M}=$ $\pi_{2} N \oplus N_{2}^{\prime}$. Set $d_{i}=\operatorname{dim}_{\mathbb{Q}} \pi_{i} N$ for $i=1$, 2. Since $N$ is invariant under the action of $s \in P_{2}$, there exists $P, Q \in G L_{2 n}(\mathbb{Q})$ such that

$$
\left(\begin{array}{cc}
P & 0 \\
0 & Q
\end{array}\right) \widehat{S}\left(\begin{array}{cc}
P^{-1} & 0 \\
0 & Q^{-1}
\end{array}\right)=\left(\begin{array}{cccc}
A & 0 & C & 0 \\
* & * & * & * \\
D & 0 & B & 0 \\
* & * & * & *
\end{array}\right)
$$

for some matrices $A, B, C, D$ of respective size $d_{1} \times d_{1}, d_{2} \times d_{2}, d_{1} \times d_{2}$, and $d_{2} \times d_{1}$. Here, $\widehat{S}$ denotes the matrix obtained from $S$ via (3).

Claim $d_{1}=d_{2}$

Since $M$ is simple, $p(s)=\operatorname{det}(S-s I)$ is irreducible in $\mathbb{Q}[s]$. In particular, $p(0)=$ $\operatorname{det}(S) \neq 0$, so $S$ is invertible. Therefore, $\left(\begin{array}{ll}0 & S \\ I & 0\end{array}\right)$ is invertible, as well as the matrix $P\left(\begin{array}{ll}0 & S \\ I & 0\end{array}\right) Q^{-1}=\left(\begin{array}{ll}C & 0 \\ * & *\end{array}\right)$. Hence, the first $d_{1}$ lines of this matrix are linearly independant. Since $C$ is of size $d_{1} \times d_{2}$, this implies that $d_{1} \leq d_{2}$. Similarly, $0 \neq p(1)=$ $\operatorname{det}(S-I)$ implies that $\left(\begin{array}{cc}-I & S \\ -I & I\end{array}\right)$ is invertible, as well as $Q\left(\begin{array}{ll}-I & S \\ -I & I\end{array}\right) P^{-1}=\left(\begin{array}{ll}D & 0 \\ * & *\end{array}\right)$. Therefore, $d_{2} \leq d_{1}$, proving the claim. This also shows that the matrices $C$ and $D$ are invertible. Note that $d:=d_{1}=d_{2}$ satisfies $0<d<2 n$, as $N \neq 0$ and $N \neq \widehat{M}$.

Using this claim and the fact that $C$ is invertible, equation (4) can be transformed into the following equalities:

$$
Q\left(\begin{array}{cc}
0 & S \\
0 & I
\end{array}\right)=\left(\begin{array}{ll}
B & 0 \\
* & *
\end{array}\right) Q, \quad Q\left(\begin{array}{ll}
I & 0 \\
I & 0
\end{array}\right)=\left(\begin{array}{ll}
E & 0 \\
* & *
\end{array}\right) Q
$$

where $E=C^{-1} A C$. This implies that $Q=\left(\begin{array}{cc}X & (E-I) X \\ * & *\end{array}\right)$ for some matrix $X$ of size $d \times n$ which satisfies the following equations:

$$
(B-I)(E-I) X=X S, \quad E(E-I) X=0, \quad B X=0 .
$$

Claim $0<\operatorname{rank} X<n$

If $r:=\operatorname{rank} X=0$, then $X=0$ so the first $d$ lines of $Q$ are zero. This is impossible since $Q$ is invertible and $d>0$. On the other hand, let us assume that $r=n$. This means that $X$ is the matrix of an injective linear map $M \hookrightarrow \mathbb{Q}^{d}$, which we also denote by $X$. Since $S$ is invertible, the first equality in (5) implies that the rank of $(E-I) X$ 
is $n$. Consider the subspace $V$ of $\mathbb{Q}^{d}$ consisting of the intersection of the images of $X$ and $(E-I) X$. Since both matrices have rank $n$ and since $d<2 n$, the space $V$ has positive dimension. Now, set

$$
W=\{w \in M \mid X w=(E-I) X m \text { for some } m \in M\} .
$$

Since $W=\{w \in M \mid X w \in V\}$ and $X$ is injective, $\operatorname{dim}_{\mathbb{Q}} W=\operatorname{dim}_{\mathbb{Q}} V>0$. Given any $w \in W$, we have $E X w=E(E-I) X m=0$ by the second equation of (5). Therefore,

$$
X S w=(B-I)(E-I) X w=-B X w+X w=X w .
$$

Since $X$ has maximal rank, this implies that $S w=w$ for all $w \in W \subset M$. As $W \neq 0$, this would imply that $M$ is not a simple $\mathbb{Q}[s]$-module. Therefore, $r<n$, proving the claim.

Let us choose two matrices $T \in G L_{d}(\mathbb{Q})$ and $U \in G L_{n}(\mathbb{Q})$ such that $T X U=$ $\left(\begin{array}{cc}I_{r} & 0 \\ 0 & 0\end{array}\right)=: \widetilde{X}$, where $I_{r}$ denotes the identity matrix of size $r=\operatorname{rank} X$. Equations (5) above give

$$
(\widetilde{B}-I)(\widetilde{E}-I) \widetilde{X}=\widetilde{X} \widetilde{S}, \quad \widetilde{B} \widetilde{X}=0,
$$

where $\widetilde{E}=T E T^{-1}, \widetilde{B}=T B T^{-1}$ and $\widetilde{S}=U^{-1} S U$. Writing $S=\left(\begin{array}{ll}S_{11} & S_{12} \\ S_{21} & S_{22}\end{array}\right)$ with $S_{11}$ and $S_{22}$ of respective size $r \times r$ and $(n-r) \times(n-r)$, these equations imply that $S_{12}=0$. Since $0<r<n, p(s)=\operatorname{det}(\widetilde{S}-s I)$ is reducible in $\mathbb{Q}[s]$, contradicting the fact that $M$ is simple. This concludes the proof of the lemma.

Lemma 2.4 Two objects $M$ and $M^{\prime}$ are isomorphic in $\mathcal{C}$ if and only if $\widehat{M}$ and $\widehat{M}^{\prime}$ are isomorphic in $\mathcal{D}$.

Proof Let $R \in G L_{n}(\mathbb{Q})$ denote the matrix of an isomorphism between $M$ and $M^{\prime}$ in $\mathcal{C}$. Then, one easily checks that the matrix $R^{\oplus 4} \in G L_{4 n}(\mathbb{Q})$ defines an isomorphism between $\widehat{M}$ and $\widehat{M}^{\prime}$ in $\mathcal{D}$. Conversely, let us assume that we have an isomorphism between $\widehat{M}$ and $\widehat{M}^{\prime}$ in $\mathcal{D}$. In particular, it is an isomorphism between the $\mathbb{Q}$-vector spaces $M^{\oplus 4}$ and $\left(M^{\prime}\right)^{\oplus 4}$. Let $H \in G L_{4 n}(\mathbb{Q})$ be a matrix of this isomorphism with respect to bases of $M^{\oplus 4}$ and $\left(M^{\prime}\right)^{\oplus 4}$ given by four copies of some fixed bases of $M$ and $M^{\prime}$. Since $h \pi_{i}=\pi_{i} h$ for $i=1,2, H$ is necessarily of the form $H=\left(\begin{array}{cc}P & 0 \\ 0 & Q\end{array}\right)$ for some $P, Q \in G L_{2 n}(\mathbb{Q})$. Furthermore, $H$ also satisfies the equation $H \widehat{S}=\widehat{S}^{\prime} H$. Here, $\widehat{S}$ (resp. $\widehat{S}^{\prime}$ ) is the matrix obtained via equation (3) from the matrix $S$ (resp. $S^{\prime}$ ) giving the action of $s \in P_{1}=\mathbb{Z}[s]$ on $M$ (resp. $M^{\prime}$ ). This implies easily that 
$P=Q=\left(\begin{array}{cc}R & 0 \\ 0 & R\end{array}\right)$ for some $R \in G L_{n}(\mathbb{Q})$ that satisfies $R S=S^{\prime} R$. This matrix $R$ defines an isomorphism in $\mathcal{C}$ between $M$ and $M^{\prime}$.

If $M$ is a $(-1)$-self-dual object in $\mathcal{C}$ via $\phi: M \rightarrow M^{*}$, then $\widehat{M}$ is a $(-1)$-self-dual object in $\mathcal{D}$ via $\widehat{\phi}: \widehat{M} \rightarrow(\widehat{M})^{*}$. Hence, Lemmas 2.3 and 2.4 imply that the Bing doubling map $(M, \phi) \mapsto(\widehat{M}, \widehat{\phi})$ induces an inclusion $\overline{\mathcal{M}}^{s}(\mathcal{C},-1) \hookrightarrow \overline{\mathcal{M}}^{s}(\mathcal{D},-1)$. By Hermitian devissage, it induces homomorphisms $\varphi_{M}$ for all $M \in \overline{\mathcal{M}}^{s}(\mathcal{C},-1)$ which fit in the following commutative diagram:

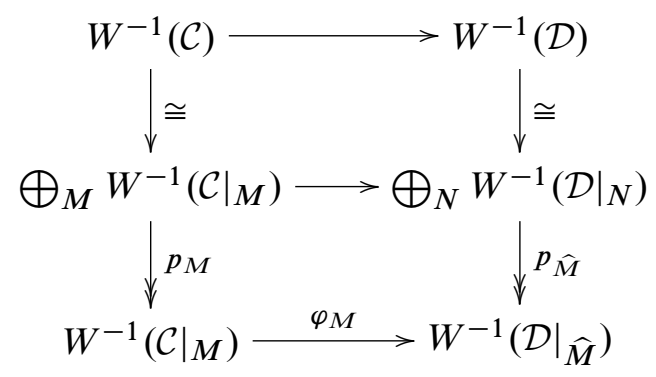

We are left with the proof that $\varphi_{M}$ is injective for all $M \in \overline{\mathcal{M}}^{S}(\mathcal{C},-1)$. Consider the additive functor $F: \mathcal{C} \rightarrow \mathcal{D}$ given by $F(M)=\widehat{M}$ and $F\left(\alpha: M \rightarrow M^{\prime}\right)=\left(\alpha^{\oplus 4}: \widehat{M} \rightarrow\right.$ $\left.\widehat{M}^{\prime}\right)$.

Lemma 2.5 If $M$ be a simple object in $\mathcal{C}$, then the ring homomorphism $\operatorname{End}_{\mathcal{C}} M \rightarrow$ $\operatorname{End}_{\mathcal{D}} \widehat{M}$ induced by $F$ is an isomorphism.

Proof A simple representation over $\mathbb{Q}$ of $P_{1}=\mathbb{Z}[s]$ is of the form $K=\mathbb{Q}[s] /(p)$, where $p \in \mathbb{Q}[s]$ is an irreducible polynomial. Therefore, $\operatorname{End}_{\mathcal{C}} M=\operatorname{End}_{\mathbb{Q}[s]} K=K$ is a field. This implies that the homomorphism induced by $F$ is injective. To check that it is onto, fix an element $\beta \in \operatorname{End}_{\mathcal{D}} \widehat{M}$. Since $\beta \pi_{i}=\pi_{i} \beta$ for $i=1,2$, a matrix for $\beta$ is necessarily of the form $\left(\begin{array}{ll}P & 0 \\ 0 & Q\end{array}\right)$ for some $P, Q \in \mathrm{M}_{2 n}(\mathbb{Q})$. As in the proof of Lemma 2.4, the equation $\beta s=s \beta$ easily implies that $P=Q=\left(\begin{array}{ll}R & 0 \\ 0 & R\end{array}\right)$ for some $R \in \mathrm{M}_{n}(\mathbb{Q})$ such that $R S=S R$. This matrix $R$ defines an element $\alpha \in \operatorname{End}_{\mathcal{C}} M$ such that $F(\alpha)=\beta$.

In particular, $F$ induces an isomorphism $F_{*}: W^{1}\left(\operatorname{End}_{\mathcal{C}} M\right) \rightarrow W^{1}\left(\operatorname{End}_{\mathcal{D}} \widehat{M}\right)$. Let $(M, b)$ be a $(-1)-$ hermitian form in $\mathcal{C}$, and let $(\widehat{M}, \widehat{b})$ be the corresponding $(-1)-$ hermitian form in $\mathcal{D}$, where a matrix for $\widehat{b}$ is obtained from a matrix for $b$ via (2). 
Using Lemma 2.5, it is routine to check that for all $M \in \overline{\mathcal{M}}^{s}(\mathcal{C},-1)$, the following diagram commutes:

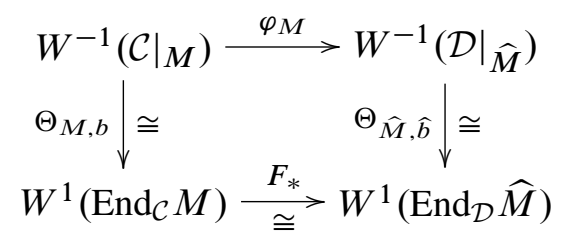

This concludes the proof of Theorem 2.1.

\section{The Rasmussen invariant}

In a beautiful paper [17], J Rasmussen used Khovanov homology to define a combinatorial invariant of smooth concordance of knots. Its extension to links was carried out by A Beliakova and S Wehrli [1]. We shall now show that this invariant, unlike the ones listed in Section 1, can tell that some Bing doubles are not smoothly slice.

Recall that the Rasmussen invariant assigns to each oriented link $L$ in $S^{3}$ an integer $s(L) \in \mathbb{Z}$. It satisfies the following properties (see [1]).

(i) If $C$ is a smooth oriented cobordism in $S^{3} \times[0,1]$ between two oriented links $L$ and $L^{\prime}$ such that every component of $C$ meets $L$, then

$$
\left|s(L)-s\left(L^{\prime}\right)\right| \leq-\chi(C) .
$$

(ii) If $L$ is represented by a positive diagram with $n$ crossings and $k$ Seifert circles, then $s(L)=n-k+1$.

(iii) If $L$ has $m$ components, then $s(L) \equiv m-1(\bmod 2)$.

Note that Property (i) implies that $s$ is an invariant of smooth concordance, and Property (ii) implies that the $s$-invariant of the $m$-component unlink is $1-m$. In particular, if $L$ is a smoothly slice $2-$ component link, then $s(L)=-1$.

Lemma 3.1 The Rasmussen invariant of a Bing double is equal to \pm 1 . 




Figure 4: A diagram $D$ for the positive trefoil knot, with the 2 northeastern corners highlighted. On this example, $\operatorname{tb}(D)=1$.

Proof The crossing change illustrated opposite turns any Bing double $B(K)$ into a positive Hopf link whose $s-$ invariant is 1 by Property (ii). This corresponds to a cobordism between $B(K)$ and the positive Hopf link with Euler characteristic -2 . By Property (i), $|s(B(K))-1| \leq 2$.
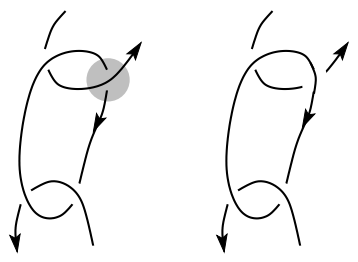
Similarly, the modification of one of the positive crossings in the diagram given here turns $B(K)$ into a negative Hopf link, whose $s$-invariant is equal to -1 . Hence, $|s(B(K))+1| \leq 2$. The lemma now follows from Property (iii).

Let us recall briefly the (combinatorial) definition of the Thurston-Bennequin invariant of a knot. We refer to Rudolph [19] for the analytic definition and further details. Every knot admits a diagram $D$ consisting of vertical and horizontal segments, the horizontal segment passing over the vertical one at each crossing. Let $\operatorname{tb}(D)$ denote the writhe of $D$ minus the number of 'northeastern corners' of $D$. (See Figure 4 for an example.) The Thurston-Bennequin invariant $\mathrm{TB}(K)$ of a knot $K$ is the maximum value of $\operatorname{tb}(D)$ over all such diagrams for $K$.

We shall be interested in knots $K$ such that $\operatorname{TB}(K) \geq 0$. Let us mention that if a non-trivial knot $K$ is strongly quasi-positive (ie, if it is the closure of the product of braids of the form $\sigma_{i j}=\left(\sigma_{i} \cdots \sigma_{j-2}\right) \sigma_{j-1}\left(\sigma_{i} \cdots \sigma_{j-2}\right)^{-1}$ with $\left.1 \leq i<j<n\right)$, then $\mathrm{TB}(K) \geq 0$. In particular, all non-trivial positive knots (non-trivial knots that admit a diagram with only positive crossings) have non-negative Thurston-Bennequin invariant.

Proposition 3.2 If a knot $K$ has non-negative Thurston-Bennequin invariant, then $s(B(K))=1$.

Proof Consider the local transformation of the Bing double illustrated in Figure 5. It describes a cobordism $C$ between the Bing double $B(K)$ and the untwisted positive Whitehead double $W h(K)$ of $K$ with $\chi(C)=-1$. Therefore, $|s(B(K))-s(W h(K))| \leq$ 1. Now, there is an obvious cobordism of Euler characteristic -1 between $W h(K)$ 


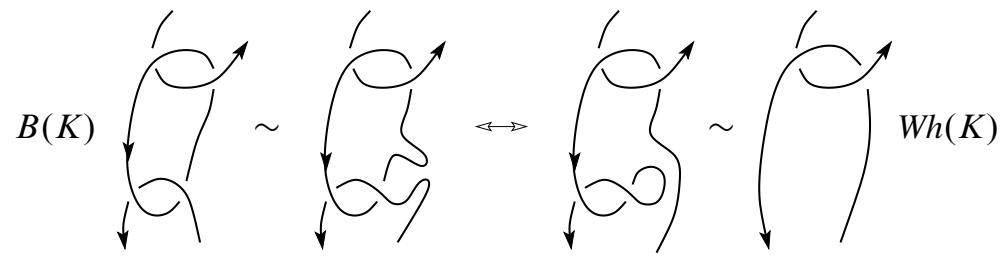

Figure 5: A cobordism between the Bing double and the untwisted positive Whitehead double of $K$

and the positive Hopf link. Hence, $|s(W h(K))-1| \leq 1$. Since $s(W h(K))$ is even, it is equal to 0 or 2 . Gathering these results with Lemma 3.1, we conclude that the only possible values of the pair $(s(B(K)), s(W h(K)))$ are $(-1,0),(1,0)$ and $(1,2)$. In particular, if $s(W h(K))=2$, then $s(B(K))=1$.

Building on ideas of L Rudolph, C Livingston showed that if a knot satisfies $\mathrm{TB}(K) \geq 0$, then $s(W h(K))=2$. This computation was originally performed for the Ozsváth-Szabó knot concordance invariant, but using only properties shared by the Rasmussen invariant (see Livingston [14, Theorem 12] and Livingston-Naik [15, Theorem 2]). Therefore, if $\mathrm{TB}(K) \geq 0$, then $s(B(K))=1$.

As an immediate consequence, we see that if $\operatorname{TB}(K) \geq 0$, then $B(K)$ is not smoothly slice. This statement is by no means new. Indeed, L Rudolph [19] showed that if $\mathrm{TB}(K) \geq 0$, then $W h(K)$ is not smoothly slice. By the cobordism illustrated in Figure 5 , this implies that $B(K)$ is not smoothly slice. However, it is interesting to see that this fact can be recovered using only basic properties of the Rasmussen invariant for links.

Proposition 3.2 also provides a wide class of links for which the Rasmussen invariant is a better obstruction to sliceness then the multivariable Levine-Tristram signature (recall Section 1). These examples are the first non-split links with this property (compare [1, Section 6.3]). Since their components are trivial, our examples also show that the obstruction to the sliceness of a link $L$ provided by $s(L)$ is more then just the obstruction to the sliceness of each components $L_{i}$ provided by $s\left(L_{i}\right)$.

One easily checks that the existence of an invariant of links that satisfies Properties (i) and (ii) above implies the slice Bennequin inequality. (The argument for knots given in Shumakovitch [21] extends to links.) By Proposition 3.2 and the end of Section 1 , we see that the Rasmussen invariant is actually stronger than the slice Bennequin inequality for detecting links that are not smoothly slice.

Let us conclude this article with one last remark. Freedman showed that the untwisted Whitehead double of any knot $K$ is topologically slice (see Freedman and Quinn 
[7]). By Corollary 1.3 , the link $B(W h(K))$ is topologically slice. On the other hand, if $\mathrm{TB}(K) \geq 0$, then $\mathrm{TB}(W h(K)) \geq 1$ by [19, Proposition 3]. By Proposition 3.2, $B(W h(K))$ is not smoothly slice. Therefore, each knot $K$ with non-negative ThurstonBennequin invariant induces a link $B(W h(K))$ that is topologically but not smoothly slice, and whose components are trivial.

Acknowledgements The author wishes to express his thanks to Mathieu Baillif, Stefan Friedl, Lee Rudolph and Stefan Wehrli. Above all, it is a pleasure to thank Peter Teichner for numerous discussions. This work was supported by the Swiss National Science Foundation.

\section{References}

[1] A Beliakova, S Wehrli, Categorification of the colored Jones polynomial and Rasmussen invariant of links arXiv:math.QA/0510382

[2] R H Bing, A homeomorphism between the 3-sphere and the sum of two solid horned spheres, Ann. of Math. (2) 56 (1952) 354-362 MR0049549

[3] R Budney, JSJ-decompositions of knot and link complements in the 3-sphere arXiv: math.GT/0506523

[4] J C Cha, K H Ko, Signature invariants of links from irregular covers and non-abelian covers, Math. Proc. Cambridge Philos. Soc. 127 (1999) 67-81 MR1692511

[5] D Cimasoni, V Florens, Generalized Seifert surfaces and signatures of colored links arXiv:math.GT/0505185

[6] D Cooper, The universal abelian cover of a link, from: "Low-dimensional topology (Bangor, 1979)", London Math. Soc. Lecture Note Ser. 48, Cambridge Univ. Press, Cambridge (1982) 51-66 MR662427

[7] M H Freedman, F Quinn, Topology of 4-manifolds, Princeton Mathematical Series 39, Princeton University Press MR1201584

[8] S Friedl, Link concordance, boundary link concordance and eta-invariants, Math. Proc. Cambridge Philos. Soc. 138 (2005) 437-460 MR2138572

[9] S Harvey, Homology cobordism invariants and the Cochran-Orr-Teichner filtration of the link concordance group arXiv:math.GT/0609378

[10] A Hatcher, Basic Topology of 3-Manifolds Available at http:// www . math. cornell. edu/ hatcher/

[11] A Kawauchi, On the Alexander polynomials of cobordant links, Osaka J. Math. 15 (1978) 151-159 MR0488022

[12] K H Ko, private communication 
[13] K H Ko, Seifert matrices and boundary link cobordisms, Trans. Amer. Math. Soc. 299 (1987) 657-681 MR869227

[14] C Livingston, Computations of the Ozsváth-Szabó knot concordance invariant, Geom. Topol. 8 (2004) 735-742 MR2057779

[15] C Livingston, S Naik, Ozsváth-Szabó and Rasmussen invariants of doubled knots, Algebr. Geom. Topol. 6 (2006) 651-657 MR2240910

[16] K Murasugi, On the Arf invariant of links, Math. Proc. Cambridge Philos. Soc. 95 (1984) 61-69 MR727081

[17] J Rasmussen, Khovanov homology and the slice genus arXiv:math.GT/0402131

[18] L Rudolph, Quasipositivity as an obstruction to sliceness, Bull. Amer. Math. Soc. (N.S.) 29 (1993) 51-59 MR1193540

[19] L Rudolph, An obstruction to sliceness via contact geometry and "classical" gauge theory, Invent. Math. 119 (1995) 155-163 MR1309974

[20] D Sheiham, Invariants of boundary link cobordism, Mem. Amer. Math. Soc. 165 (2003) x+110 MR1997849

[21] A Shumakovitch, Rasmussen invariant, Slice-Bennequin inequality, and sliceness of knots arXiv:math.GT/0411643

[22] P Teichner, private communication

Department of Mathematics, UC Berkeley, 970 Evans Hall

Berkeley, CA 94720, USA

cimasoni@math. berkeley.edu

Received: 16 September 2006 